\title{
Exames imaginológicos realizados na Clínica Odontológica de uma Universidade Pública do Nordeste do Brasil: levantamento das principais indicações
}

Imaging exams performed at the Dental Clinic of a Public University in Northeastern Brazil: survey of the main indications

Exámenes de imagen realizados en la Clínica Dental de una Universidad Pública del Noreste de Brasil: encuesta de las principales indicaciones

\author{
Ana Cecília Carenina Machado Mourão \\ ORCID: https://orcid.org/0000-0002-8569-224X \\ Universidade Federal do Ceará, Brasil \\ E-mail: ceciliamachadoodontologia@gmail.com \\ Antônio Odacy Souza \\ ORCID: https://orcid.org/0000-0003-4854-806X \\ Universidade Federal do Ceará, Brasil \\ E-mail: odacyfortaleza@gmail.com \\ Filipe Nobre Chaves \\ ORCID: https://orcid.org/0000-0001-6345-8156 \\ Universidade Federal do Ceará, Brasil \\ E-mail: filipenobrechaves@gmail.com \\ Denise Helen Imaculada Pereira de Oliveira \\ ORCID: https://orcid.org/0000-0001-6020-2374 \\ Universidade Federal do Ceará, Brasil \\ E-mail: denisehelen2011@hotmail.com \\ Marcelo Bonifácio da Silva Sampieri \\ ORCID: https://orcid.org/0000-0001-7942-6642 \\ Universidade Federal do Ceará, Brasil \\ E-mail: mar_sampieri@hotmail.com
}

\begin{abstract}
Resumo
Exames imaginológicos aliados aos aspectos clínicos são de suma importância para o correto diagnóstico e tratamento para o caso problema vivido pelos pacientes que procuram os serviços de saúde. Dentre os exames podemos citar a Radiografia Panorâmica (RP) e a Tomografia Computadorizada de Feixe Cônico (TCFC) que apresentam diversas indicações na área odontológica. O objetivo desse trabalho foi realizar uma análise das principais indicações para a realização desses exames. As solicitações foram gentilmente cedidas pela Universidade Federal do Ceará - Campus Sobral totalizando 252 RPs e 186 TCFCs realizadas no período de agosto a outubro de 2019. As indicações foram organizadas em nove grupos: ATM, Cirurgia, Endodontia, Estomatologia, Implantodontia, Odontopediatria, Periodontia, Outras Indicações e Sem indicações. De acordo com os resultados percebemos que o maior número de indicações das RPs foi na Cirurgia destacando a avaliação de terceiros molares inclusos, enquanto que a principal indicação das TCFCs foi para a implantodontia destacando-se o planejamento pré-operatório para colocação de implantes. Vale ressaltar ainda, a alta prevalência de solicitações de exames sem indicação descrita. Portanto, concluise que necessita de uma educação no âmbito universitário para o correto preenchimento das fichas de solicitação além do estabelecimento de um protocolo clínico para padronizar tais indicações de forma a aperfeiçoar a documentação dos casos. Além disso, qualquer que seja o exame de imagem escolhido para o caso problema em questão, deve sempre ser levada em consideração a sua necessidade, a fim de não expor o paciente à dose de radiação desnecessária. Palavras-chave: Radiologia; Epidemiologia; Radiografia panorâmica; Tomografia computadorizada de feixe cônico.
\end{abstract}

\begin{abstract}
Imaging exams combined with clinical aspects are of paramount importance for the correct diagnosis and treatment of the problem case experienced by patients who seek health services. Among the exams we can mention the Panoramic Radiography (PR) and the Cone Beam Computed Tomography (CBCT), which have several indications in the dental field. The objective of this work was to analyze the main indications for carrying out these tests. The requests were kindly granted by the Federal University of Ceará - Campus Sobral, totaling 252 PRs and 186 CBCTs carried out from August to October 2019. The indications were organized into nine groups: ATM, Surgery, Endodontics, Stomatology, Implantology, Pediatric Dentistry, Periodontics, Other Indications and No Indications. According to the results, we noticed that the greatest number of indications for PRs was in Surgery, highlighting the evaluation of
\end{abstract}


impacted third molars, while the main indication of CBCTs was for implant dentistry, highlighting the preoperative planning for implant placement. It is also worth mentioning the high prevalence of exam requests without a described indication. Therefore, it is concluded that there is a need for education at the university level to correctly fill out the application forms, in addition to establishing a clinical protocol to standardize such indications in order to improve the documentation of cases. In addition, whatever the imaging exam chosen for the problem case in question, its need must always be taken into account, in order not to expose the patient to unnecessary radiation dose.

Keyworlds: Radiology; Epidemiology; Radiography panoramic; Cone-beam computed tomography.

\begin{abstract}
Resumen
Los exámenes de imagen combinados con los aspectos clínicos son de suma importancia para el correcto diagnóstico y tratamiento del caso problema experimentado por los pacientes que buscan servicios de salud. Entre los exámenes podemos mencionar la Radiografía Panorámica (RP) y la Tomografía Computarizada Cone Beam (CBCT), que tienen varias indicaciones en el campo dental. El objetivo de este trabajo fue analizar las principales indicaciones para la realización de estas pruebas. Las solicitudes fueron atendidas amablemente por la Universidad Federal de Ceará Campus Sobral, por un total de 252 PR y 186 TCFC realizadas de agosto a octubre de 2019. Las indicaciones se organizaron en nueve grupos: ATM, Cirugía, Endodoncia, Estomatología, Implantología, Odontopediatría, Periodoncia, Otras Indicaciones y Sin Indicaciones. De acuerdo con los resultados, notamos que el mayor número de indicaciones de PR fue en Cirugía, destacando la evaluación de terceros molares impactados, mientras que la principal indicación de CBCT fue para implantología, destacando la planificación preoperatoria para la colocación de implantes. También vale la pena mencionar la alta prevalencia de solicitudes de exámenes sin una indicación descrita. Por tanto, se concluye que existe una necesidad de formación a nivel universitario para cumplimentar correctamente los formularios de solicitud, además de establecer un protocolo clínico para estandarizar dichas indicaciones con el fin de mejorar la documentación de los casos. Además, sea cual sea el examen de imagen elegido para el caso problemático en cuestión, siempre se debe tener en cuenta su necesidad, para no exponer al paciente a dosis de radiación innecesarias.
\end{abstract}

Palabras clave: Radiología; Epidemiología; Radiografía panorámica; Tomografía computarizada de haz cónico.

\title{
1. Introdução
}

“A luz nova vê os ossos através da carne!" Foi assim que foi dada a notícia para o mundo em meados de 1896, no Jornal Viena Presse, da descoberta os raios X. Desde sua descoberta, que rendeu o primeiro Prêmio Nobel de Física em 1901 a Röetgen, até os dias atuais a importância dos raios X como ferramenta auxiliar de diagnóstico nas diversas áreas da saúde tem sido inquestionável (Francisco et al., 2005) e suas limitações têm sido superadas com o desenvolvimento tecnológico (Gustavo et al., 2010).

Segundo Tavano e Alvares (2002) citado por Haas (2013, p.10) na odontologia, seu uso foi iniciado em 1898 pelo Dr. Otto Walkhoff que fez a primeira radiografia dental. Mas foi apenas em 1899, através de Edmund Kells em que houve os primeiros sinais de uma padronização para a realização dos exames radiológicos (Freitas et al., 2000). Os raios X ocasionaram uma verdadeira revolução na saúde como um todo e seu uso dentro da Odontologia tem se desenvolvido e modificado ao longo dos anos, culminando na Radiografia Panorâmica em 1949 (Freitas et al., 2000), e mais recentemente no desenvolvimento do aparelho de Tomografia Computadorizada de Feixe Cônico (TCFC) em 1998 (Mozzo et al., 1998).

Segundo White SC, Pharoah MJ (2007, p. 210) a Radiografia Panorâmica (RP)

[...] se baseia no princípio de movimento recíproco da fonte de raios $\mathrm{X}$ e de um receptor da imagem ao redor de um plano ou ponto central, denominado plano de corte, no qual se localiza o objeto de interesse. Objetos em frente ou atrás desse plano de corte não são visibilizados de forma clara em virtude do seu movimento em relação ao centro de rotação do receptor e da fonte de raios X.

Segundo Whaites (2011), esta se tornou uma técnica bastante popular na Odontologia por apresentar diversas vantagens como o fato de todos os dentes e suas estruturas de suporte serem mostrados em uma única imagem. A simplicidade da técnica também pode ser considerada um das vantagens, além da baixa dose de radiação (Alves, 2002; Gartner \& Goldenberg, 2009) - cerca de um quinto da dose de um exame periapical completo com filmes intraorais. Além de permitir a 
visualização da estrutura maxilo mandibular em uma única imagem, esta técnica também inclui as estruturas circunvizinhas, como a maxila, fossa nasal, articulação têmporo-mandibular, processo estilóide, osso ióide e espaços aéreos oro-faringeanos (Alves, 2002). White SC, Pharoah MJ (2007) acrescentam ainda que essa técnica não causa desconforto podendo ser utilizada inclusive em pacientes com dificuldade de abertura bucal e com curto tempo necessário para sua realização (3 a 4 minutos).

Como desvantagens podemos citar que as técnicas radiográficas convencionais, dentre elas a panorâmica, oferecem imagens limitadas a duas dimensões, e estruturas complexas e tridimensionais são sobrepostas e visualizadas em apenas dois planos. Além da sobreposição de imagens, é também frequente a ocorrência de distorções geométricas e volumétricas dos dentes e reparos anatômicos investigados (Gustavo et al., 2010; White \& Pharoah, 2004). Outrossim o fato de ser apenas uma área, ou seja uma área focal, estruturas ou anormalidades fora dessa área poderiam passar despercebidas. Outra desvantagem seria a sobreposição de estruturas como a coluna vertebral, por exemplo, podendo levar a uma dificuldade na interpretação da imagem (Whaites, 2009). As radiografias panorâmicas não apresentam o detalhamento nem a mesma qualidade das radiografias intra-orais e periapicais (Alves, 2002; White \& Pharoah, 2004) devido á distância foco filme fixa, à distância objeto filme variável por causa de mandíbulas e dentes de tamanhos diferentes, à disposição dos dentes nos arcos dentários e à assimetria da maxila e mandíbula (Alves, 2002). Portanto, as radiografias panorâmicas são indicadas quando não é requerido a alta definição e o alto detalhamento das radiografias intra-orais.

Como uma evolução dos exames de Raio-X surge a tomografia em 1970 e desde então evoluiu até chegar a tomografia computadorizada de feixe cônico (Garib et al., 2007). A tecnologia da Tomografia Computadorizada de Feixe Cônico surge em 1998 com Mozzo e colaboradores, na Universidade de Verona (Garib et al., 2007) batizada na época como NewTom-9000, aumentando consideravelmente a capacidade de diagnóstico e planejamento odontológico (Gustavo et al., 2010). A técnica de feixe cônico já havia sido utilizada anteriormente, mas para fins distintos do odontológico como radioterapia, imaginologia vascular e microtomografia de pequenos espécimes com aplicabilidade biomédica ou industrial (Garib et al., 2007). A tomografia é conceituada segundo White SC, Pharoah MJ (2007) como "uma técnica especializada que produz radiografias mostrando somente um corte ou lâmina do paciente". Os cortes tomográficos são metaforicamente descritos como "fatias de um pão de forma" em que cada fatia representa um plano focal, ou camada focal (Carraro \& Santos, 2014; Garib et al., 2007). Ao obter fatias, a informação tridimensional também pode ser obtida imagem (Whaites, 2009). Em relação a dose de radiação, as tomografias computadorizadas convencionais são indiscutivelmente superiores as radiografias convencionais. Contudo, a dose de radiação da tomografia computadorizada de feixe cônico tem se mostrado significativamente inferior a tomografia computadorizada convencional - cerca de 1/6 desta (Garib et al., 2007). Quando se compara com as radiografias convencionais ela é semelhante à do exame periapical de boca inteira ou o equivalente a 4 a 15 vezes a dose de uma radiografia panorâmica (Carraro \& Santos, 2014; Garib et al., 2007).

As principais vantagens da aquisição da imagem tomográfica pela tecnologia de feixe cônico são: 1) Reconstrução direta dos pontos captados por reconstruções axiais, coronais e sagitais sem reformatação; 2) Sofisticação tecnológica, em que a velocidade da totalidade do corte é controlada através de um programa eletrônico e não, por velocidade do tubo de raios X; 3 ) Mesmas condições de tempo de escaneamento, através de uma simples aquisição, diminuindo, sobremaneira, a dose de radiação e dispensando o mecanismo de cortes (Garib et al., 2007; Gustavo et al., 2010). Existe ainda a possibilidade de impressão dos exames podendo anexá-los ao prontuário dos pacientes (Garib et al., 2007). Outrossim, quando comparamos esse tipo de exame com a Tomografia Computadorizada Convencional ("Tomografia Médica") podemos citar como principais vantagens a redução de custos, a redução da radiação, o menor tempo de exposição do paciente a radiação e a menor quantidade de artefatos produzidos na presença de metais (Garib et al., 2007). É, portanto um método não invasivo, rápido e de alta definição (Carraro \& Santos, 2014). 
Como desvantagem apresenta o custo que ainda é relativamente alto se comparado a RP, embora ela tenha adentrado cada vez mais na prática clínica como um exame complementar padrão-ouro na Odontologia.

O presente trabalho tem por objetivo fazer um levantamento das solicitações de exames de Tomografia computadorizada de feixe cônico e Radiografia Panorâmica realizadas na Universidade Federal do Ceará - Campus Sobral desde abril de 2019 a novembro de 2019 e analisar, de acordo com a literatura, se estão sendo corretamente indicados. Outrossim tem o objetivo de elaborar uma nova folha de solicitação desses exames que supra a necessidade de informações de forma a colaborar com o planejamento e acompanhamento dos pacientes.

\section{Metodologia}

O estudo se caracterizou, de acordo com Estrela (2018), como retrospectivo, quantitativo, epidemiológico com análise de prontuários, sem intervenção direta dos pacientes da pesquisa. Toda a metodologia de pesquisa foi aprovada pelo Comitê de Ética em Pesquisa (processo 4.006.660). Foi realizado um levantamento de solicitações de radiografias panorâmicas e tomografias computadorizadas de feixe cônico realizados na Universidade Federal do Ceará - Campus Sobral no período de abril de 2019 a novembro de 2019 obtendo um total de 438 solicitações de exames.

Os dados foram tabelados em forma de planilha no programa Microsoft Excel 2010 e posteriormente organizados na forma de gráficos com números absolutos e percentuais das indicações. As indicações por sua vez foram classificadas em nove grandes grupos: ATM, Cirurgia, Endodontia, Estomatologia, Implantodontia, Odontopediatria, Periodontia, Outras Indicações e Sem indicações. Esses dois últimos foram utilizados para identificar aquelas solicitações que não se encaixaram dentro dos primeiros sete grupos e que não apresentaram indicação expressamente escrita na solicitação do exame, respectivamente. Após este levantamento foi elaborada uma nova ficha de requisição de exames imaginológicos para uso da Clínica de Odontologia da Universidade Federal do Ceará - Campus Sobral.

\section{Obtenção das solicitações}

Os exames de radiografia foram feitos com o aparelho (Figura 1) Eagle 3D da empresa DABI ATLANTE $®$ acoplado a tecnologia Vbeam e até 8 volumes de campo de visão (desde $5 \times 5$ até $23 \times 16$ ) e as solicitações foram obtidas com a universidade. Os critérios de inclusão foram os prontuários de pacientes que tenham solicitações de exame de imagem, devidamente preenchidas, sem rasuras, com letra legível nos quais foi possível determinar a indicação do exame. E os critérios de exclusão foram os prontuários de pacientes que tenham solicitações de exame de imagem indevidamente preenchidas, com rasuras nos quais não é possível determinar a indicação do exame. 
Figura 1: Aparelho de Radiografia Panorâmica da Universidade Federal do Ceará - Campus Sobral.

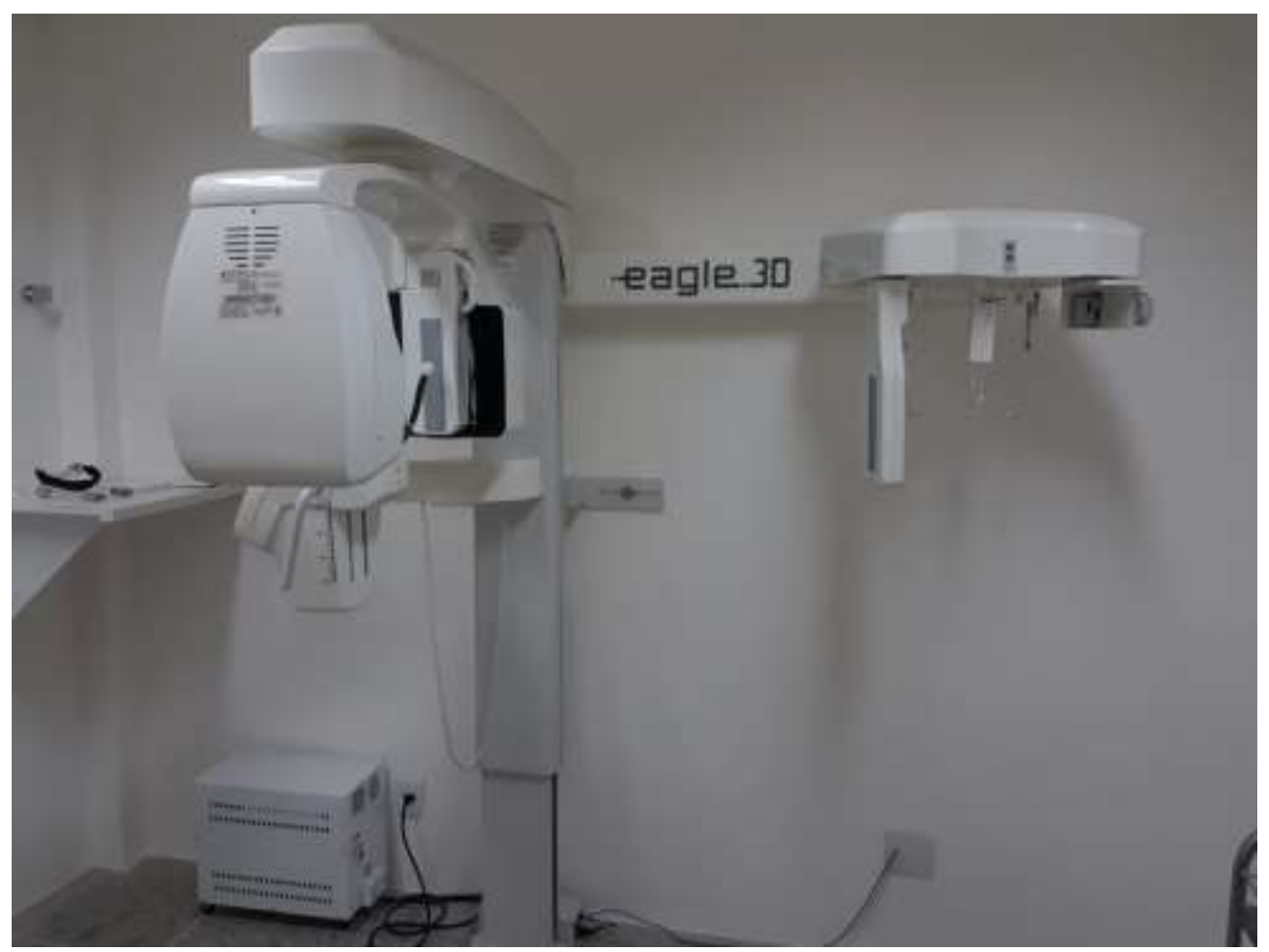

Fonte: Autores.

\section{Resultados}

Foram realizados 438 exames imaginológicos no período de abril a primeiro de novembro de 2019. Dentre estes, 252 tratavam-se de radiografias panorâmicas e 186 tratavam-se de tomografias computadorizadas de feixe cônico. Os dados foram organizados em dois quadros e dois gráficos, sendo os dois primeiros relacionados aos dados em número absolutos e os dois últimos relacionados às informações em porcentagem.

Na Tabela 1 temos que em números absolutos a principal indicação para a realização da radiografia panorâmica foi a cirurgia, mais especificamente a exodontia de terceiros molares com 115 indicações seguido pelo grupo de sem indicações com 80 solicitações, outras indicações com 24 , odontopediatria com 15 , estomatologia com 7 , periodontia com 5 , implatodontia com 3, endodontia com 2 e ATM com apenas uma indicação. Fato é que a cirurgia se mostrou como o primeiro lugar absoluto entre as indicações, corroborando com os achados no Gráfico $1 \mathrm{em}$ que esta sozinha representa mais $46 \%$ de todas as solicitações. 
Tabela 1: Indicações em números absolutos de Radiografias Panorâmicas.

\begin{tabular}{lcc}
\hline & INDICAÇÃO & QUANTIDADE DE SOLICITAÇÕES \\
\hline Cirurgia & 115 \\
Odontopediatria & 15 \\
Estomatologia & 7 \\
Periodontia & 5 \\
Implantodontia & 3 \\
Endodontia & 2 \\
ATM & 1 \\
Sem indicação & 80 \\
Outras indicações & 24 \\
\hline
\end{tabular}

Fonte: Autores.

Gráfico 1: Porcentagem de indicações de radiografias panorâmicas.

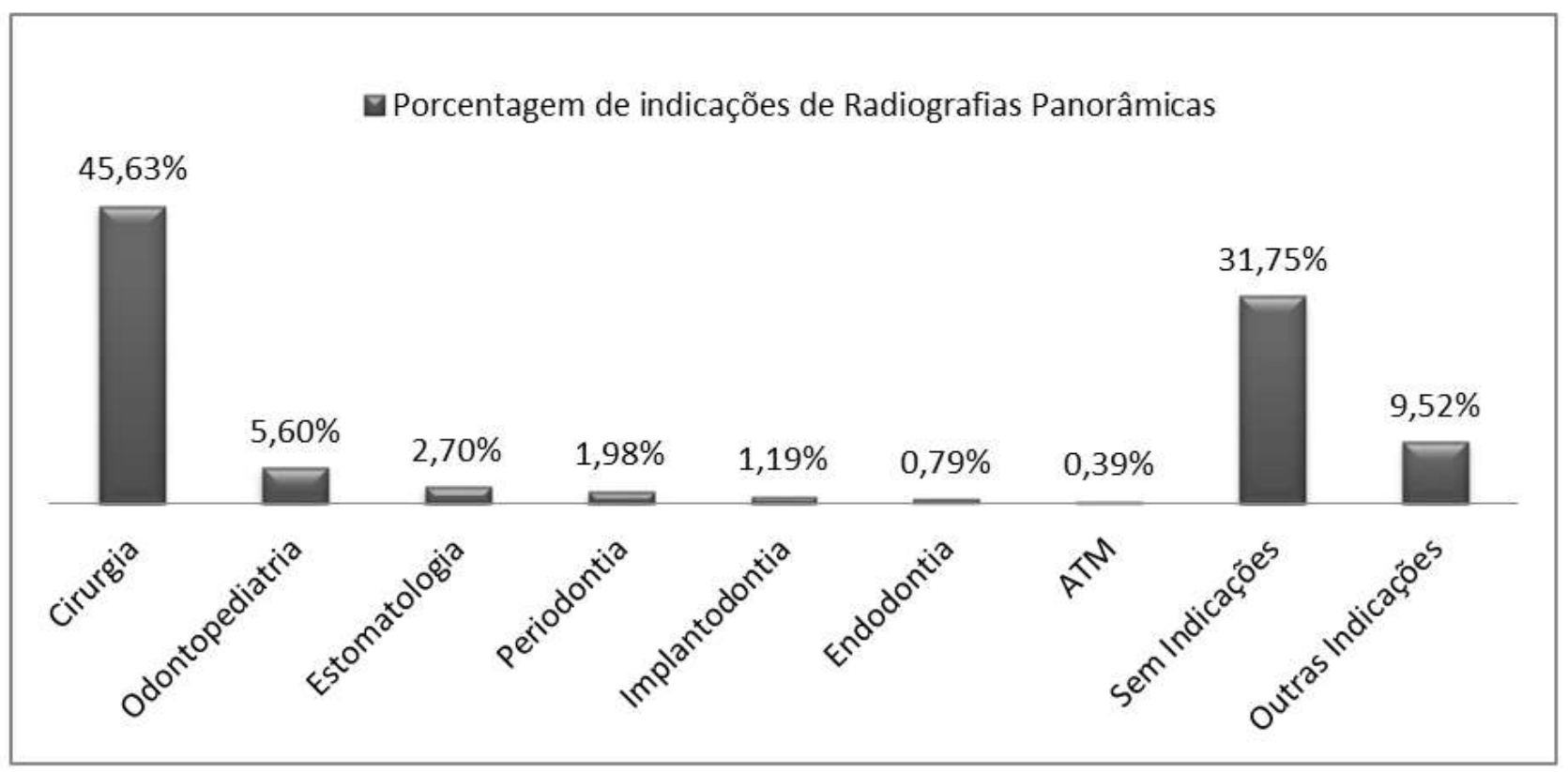

Fonte: Autores.

Na Tabela 2 em que é mostrada as principais indicações em números absolutos das tomografias computadorizadas de feixe cônico podemos perceber que a principal indicação é a implantodontia com 55 solicitações, seguido pelo grupo de sem indicações com 51 solicitações, Endodontia com 26, ATM com 10, Estomatologia com 8, outras indicações com 4 e pediatria e periodontia ambas com 2 solicitações cada. No Gráfico 2, verificamos a prevalência da implantodontia dentre as solicitações de exames representando cerca de $30 \%$ sendo seguida pela cirurgia com $15 \%$. 
Tabela 2: Indicações em números absolutos de Tomografias Computadorizadas de Feixe Cônico.

\begin{tabular}{lc}
\hline \multicolumn{1}{c}{ INDICAÇÃO } & QUANTIDADE DE SOLICITAÇÕES \\
\hline Cirurgia & 27 \\
Odontopediatria & 2 \\
Estomatologia & 8 \\
Periodontia & 2 \\
Implantodontia & 55 \\
Endodontia & 26 \\
ATM & 10 \\
Sem indicação & 51 \\
Outras indicações & 4 \\
\hline
\end{tabular}

Fonte: Autores.

Gráfico 2: Porcentagem de indicações de tomografias computadorizadas de feixe cônico.

Porcentagem de indicações de Tomografias Computadorizadas de Feixe Cônico

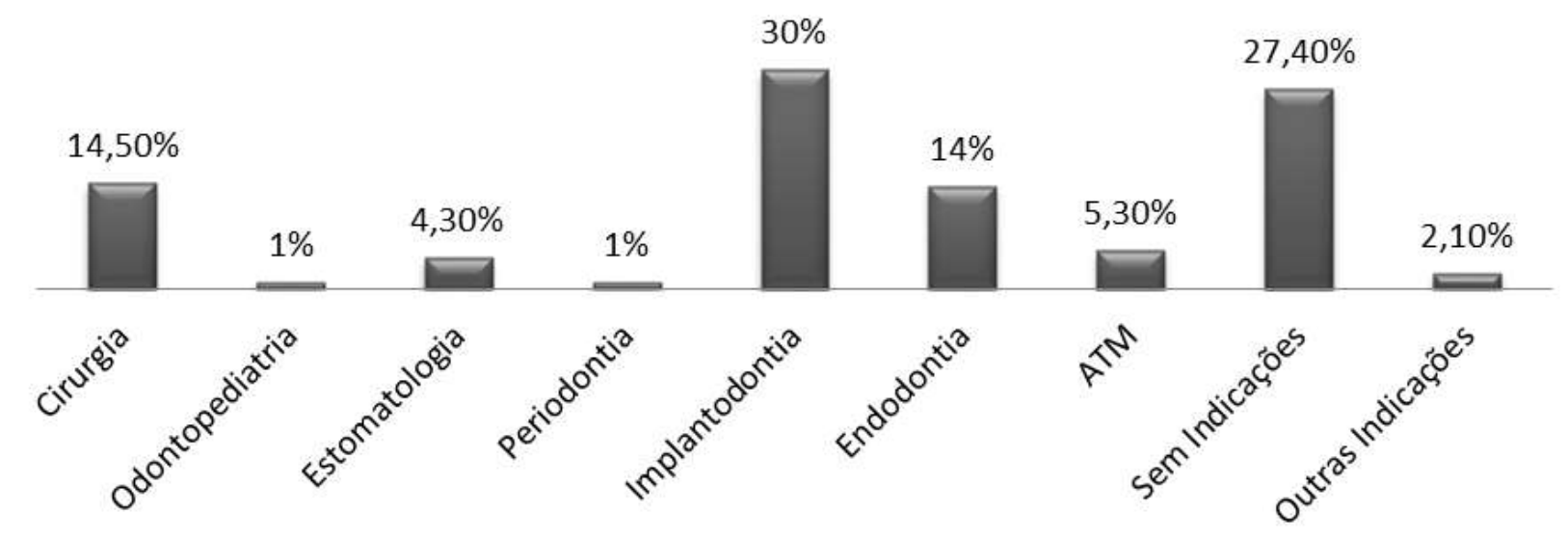

Fonte: Autores.

\section{Discussão}

Segundo o levantamento realizado no presente trabalho, pode-se observar que as principais indicações da Radiografia Panorâmica (RP) foram aos campos da cirurgia, outras indicações e odontopediatria respectivamente. De acordo com as referências bibliográficas estabeleceu-se que as principais indicações deste exame complementar seriam quando não é requerido a alta definição e o alto detalhamento das radiografias intra-orais como, por exemplo, na avaliação de traumatismo, localização de terceiros molares, patologias extensas, lesões grandes conhecidas ou suspeitas, desenvolvimento dentário (em especial na dentição mista), retenção de dentes ou ápices radiculares (em pacientes edêntulos) e anomalias de desenvolvimento (White \& Pharoah, 2004).

Acrescenta-se ainda que radiografia panorâmica ainda é muito utilizada segundo pesquisa realizada por Rushton, Homer, Worthington (1999) para: a) planejamento de cirurgia oral (95\%); b) trauma facial (90\%); c) doença periodontal (87,8\%); d) dentição muito restaurada (84,2\%); e) primeiro atendimento ao paciente (79\%) (Alves, 2002).

O maior número de indicações da Radiografia Panorâmica na pesquisa realizada por este trabalho está relacionado à cirurgia, mais especificamente ao planejamento de exodontias de terceiros molares (Figura 2). Os terceiros molares são dentes 
que apresentam uma anatomia complexa, por vezes misturando características dos primeiros e segundos molares. Além da morfologia, sua posição vestíbulo-lingual, horizontal e mésio-distal são variadas segundo a literatura o que dificulta a realização do procedimento com segurança e redução de riscos sem a realização de um exame imaginológico prévio. Segundo Silva, Passos (2014) a exodontia de terceiros molares é um procedimento que pode acarretar sérias complicações ao paciente como: hemorragia, alveolite, infecção, fratura radicular, fratura mandibular e lesão aos dentes vizinhos (Kato et al., 2016). Por isso, a RP fornece algumas informações importantes para a extração dos terceiros molares inferiores como o grau de formação e erupção, idade dentária, presença ou ausência destes, posição dentária bidimensional, proximidade com dentes adjacentes, proximidade com nervo alveolar inferior e a própria simetria mandibular. A verificação da proximidade das raízes de terceiros molares com o canal mandibular é inclusive uma de suas principais utilizações, podendo verificar através deste exame diversos sinais sugestivos tais como a interrupção da parede do canal mandibular, obscurecimento dos ápices, desvio do canal mandibular e estreitamento dos ápices (Ghaeminia et al., 2009, 2011). Contudo, em uma pesquisa concluiu-se que a TCFC foi superior à radiografia panorâmica na previsão de exposição neurovascular pós extração de terceiros molares fazendo deste tipo de exame o padrão-ouro na determinação da relação entre terceiro molar inferior e canal mandibular (da Silveira et al., 2016).

Figura 2: Radiografia Panorâmica realizada na Universidade Federal do Ceará - Campus Sobral.

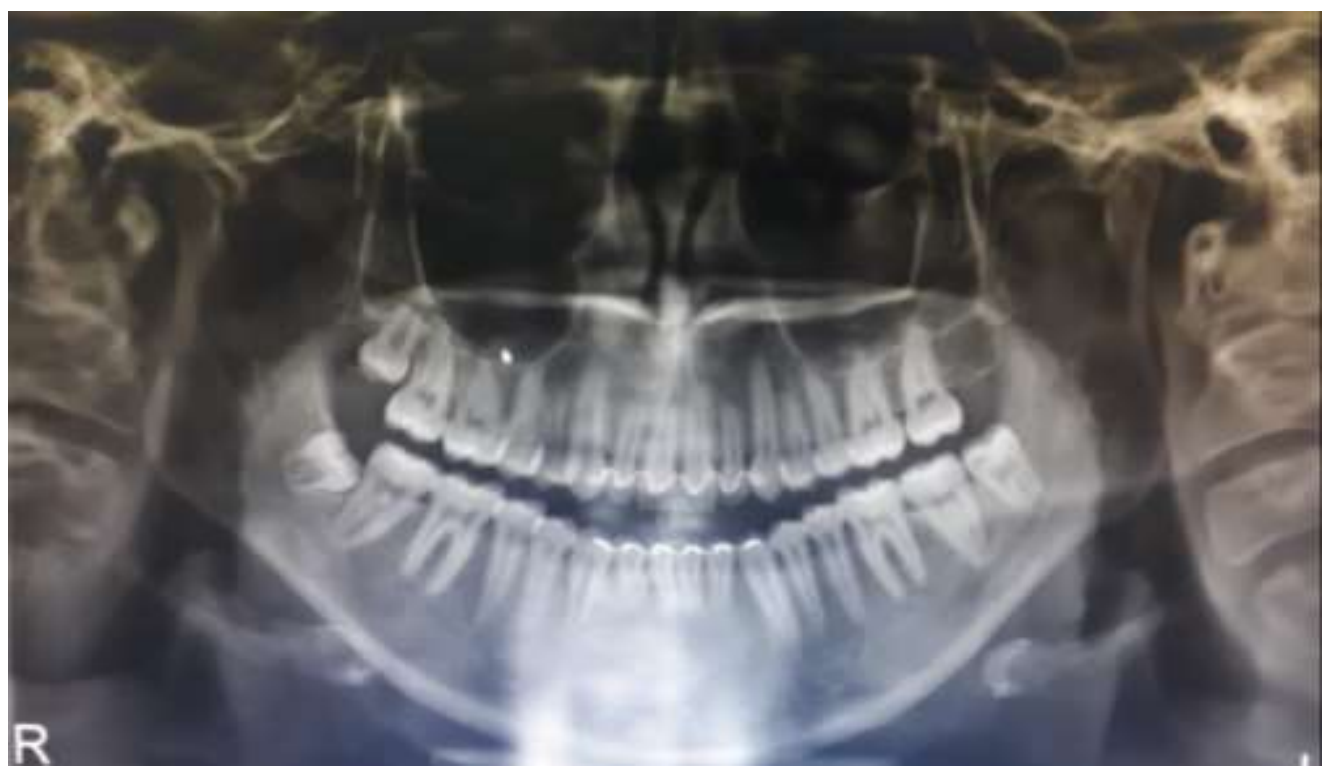

Fonte: Estomatologia UFC - Sobral.

Em relação às outras indicações, o segundo grupo com maior número de indicações, foi a utilização de radiografias panorâmicas para a verificação da presença de alongamentos no processo estilo-hioideo. Segundo estudo feito por nunes, Mourão, et al. (2020) a prevalência do alongamento do processo estilo-hioideo, que pode levar ao desenvolvimento da chamada Síndrome de Eagle, foi de 46,2\%. A síndrome de Eagle ocorre da associação de um processo estiloideo alongado (superior a $30 \mathrm{~mm}$ ) com outros sintomas como dor de cabeça, dores ao virar a cabeça, otalgia, entre outras. Esse alongamento pode levar inclusive à compressão da artéria carótida consequentemente levando a um AVC segundo relato de caso (Jelodar et al., 2018). Daí a importância das medições que podem ser simples e realizadas nas radiografias panorâmicas.

Em terceiro lugar em relação à radiografia panorâmica, foi observada a odontopediatria com uma totalidade de 15 indicações. A radiografia panorâmica é bem indicada nesse grupo dada às diversas informações que podem ser obtidas, facilidade da técnica, visão de conjunto maxilo-mandibular e a sua aceitação pelas crianças, por ser uma técnica extrabucal. Entre as informações relacionadas a essa área que podem ser obtidas através da RP temos a avaliação da dentadura mista, 
momento em que se origina a maioria das maloclusões; realização da supervisão de espaços; perda precoce de dentes decíduos; presença de diastemas; impactação de caninos; anomalias de desenvolvimento e de erupção como alterações na sequência eruptiva. Essas últimas, muitas vezes passam despercebidas no exame clínico e podem ser visualizadas precocemente na RP. A visualização de extensas patologias também podem ser observadas, bem como sinusites, comunicações buco sinusais e traumas dos maxilares (Gartner \& Goldenberg, 2009; Nonato, 2006). Dentre as principais patologias que podem ser visualizadas temos os cistos e tumores odontogênicos. A frequência de cistos e tumores nas crianças é relativamente baixa, mas quando ocorrem estão geralmente associados a impaccão de dentes permanentes. Um exemplo disso, é o odontoma, composto (tumor misto de origem epitelial e mesenquimal) que apresenta-se como uma massa de dentículos sendo considerados uma barreira física para a erupção dos dentes permanentes, principalmente os anteriores superiores. Retenção prolongada de dentes decíduos, apinhamento e hiperplasia gengival são consideradas consequências da impacção dentária (Almeida et al., 2015; Borsatto \& Nelson-filho, 2007; Dalla et al., 2016; Pozzer et al., 2009).

Além do levantamento de solicitações de RPs, foi realizado também o levantamento das Tomografias Computadorizadas de Feixe Cônico (TCFCs) realizadas. Suas indicações são amplas, mas Whaites (2007) as descreve como indicações as seguintes: avaliação nas três dimensões dos maxilares para colocação de implantes bem como sua avaliação pósoperatória, avaliação do seio maxilar, avaliação de fraturas faciais e avaliação da ATM (Whaites, 2007, Gustavo, Rodrigues, $e t$ al., 2010). As TCFCs ainda podem ser utilizadas na periodontia; endodontia; ortodontia; odontopediatria; pacientes especiais e fissurados. Esse tipo de tecnologia é utilizado inclusive para a confecção de guias cirúrgicos e auxiliam o planejamento cirúrgico e protético dentro da implantodontia (Carraro \& Santos, 2014; Gustavo et al., 2010).

Considerando os dados encontrados na presente pesquisa temos que a principal indicação dos exames tomográficos seria no grupo da implantodontia. Segundo os estudos realizados por carraro, Santos (2014) a tomografia computadorizada é imprescindível para o planejamento de implantes dentários, durante a cirurgia com a utilização de guias cirúrgicos e na reabilitação protética também (Figura 1). O artigo também conclui que esse exame em relação à radiografia panorâmica apresenta maior confiabilidade em relação às dimensões e a possibilidade de medir a espessura da cortical óssea onde será colocado o implante dentário (Carraro \& Santos, 2014). Em relação também as medidas, a TCFC eliminou as distorções, obtendo-se assim o tamanho real do objeto (Borges \& Lobo, 2016). 
Figura 3: Software utilizado na UFC - Campus Sobral para a colocação de implantes.

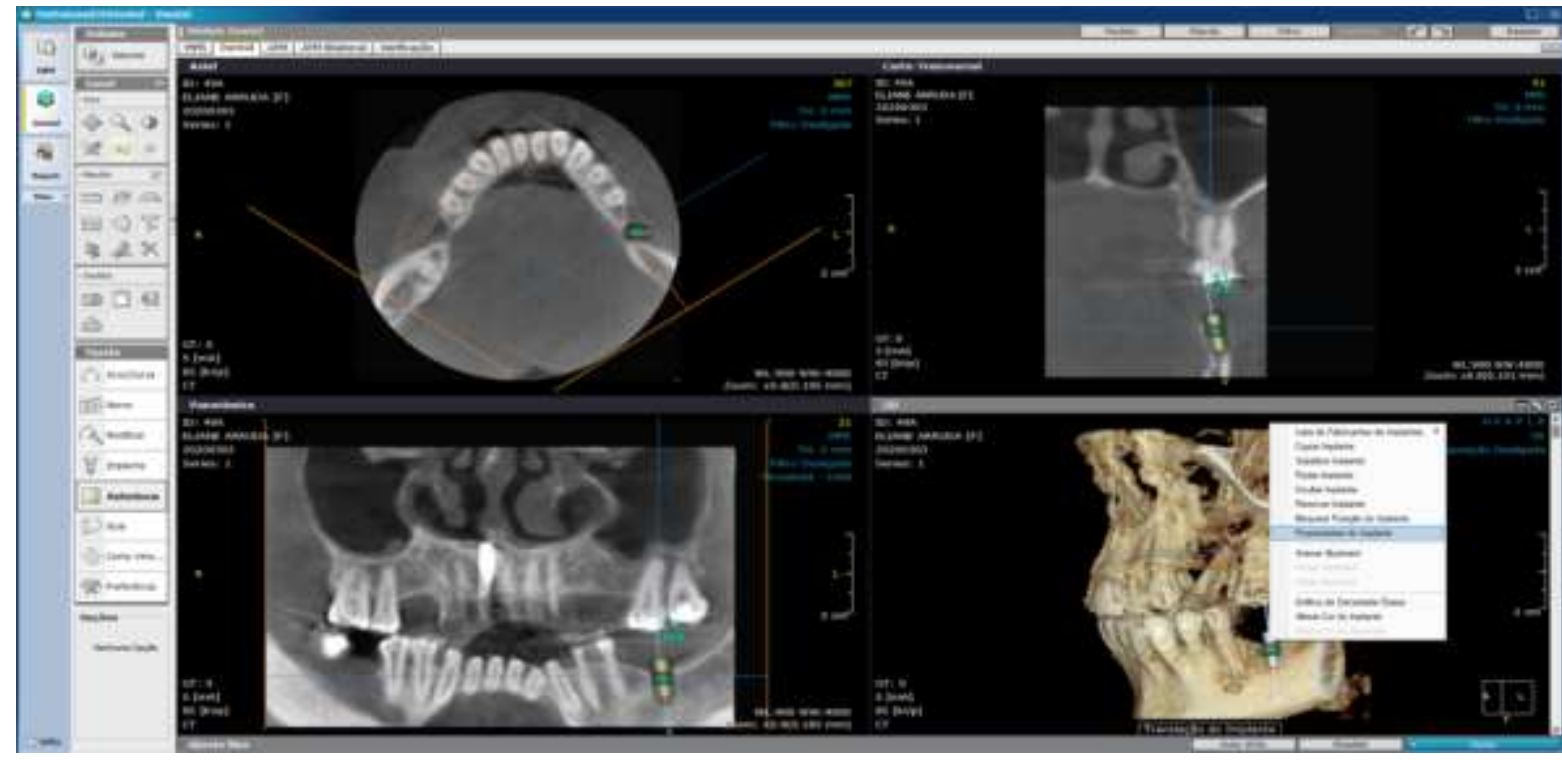

Fonte: Estomatologia UFC - Sobral.

Em segundo lugar em relação às indicações temos a cirurgia com 15\% do total de solicitações de TCFCs. Podemos citar nesse caso a especificidade da indicação na cirurgia de exodontia de terceiros molares. Como já foi citado, sabe-se que a radiografia panorâmica é importante e na maioria dos casos suficiente para determinar a posição desses dentes em relação às estruturas anatômicas adjacentes. Contudo, quando se analisa sinais radiográficos de proximidade do canal mandíbula com os terceiros molares inferiores como, por exemplo, a não visualização do ápice do terceiro molar que desaparece em meio ao canal mandibular, liga-se o sinal de alerta. A radiografia panorâmica sozinha nesses casos não é mais suficiente para garantir uma segurança no procedimento evitando complicações. Segundo o estudo em questão feito por Santos et all (2016), em 65,8\% dos casos de terceiros molares vistos na RP, a distância entre o canal mandibular e os terceiros molares inferiores era nula. Isso significa que nesses casos as estruturas estão em contato representando um risco de parestesia pós-operatória e justificando a utilização da TCFC (Guilherme et al., 2016). A precisão da TCFC para a visualização das raízes desses dentes e sua proximidade com o nervo (FIGURA 2) é relatada no estudo feito por Pippi $e t$. Al, 2016, em que em todos os casos em que foi observado uma exposição do nervo, igualmente na imagem tomográfica era visualizado a descontinuidade da cortical (Pippi et al., 2016).

Além disso, em casos em que na RP a imagem seria classificada como de alto risco para exposição do nervo alveolar inferior, quando visualizada na TCFC foram reclassificadas como de baixo risco (Ghaeminia et al., 2011). Outrossim, o próprio número de raízes foi diferenciado nos dois tipos de técnicas (Suomalainen et al., 2010). 
Figura 4: Software utilizado na UFC - Campus Sobral para a visualização da proximidade com o canal mandibular.

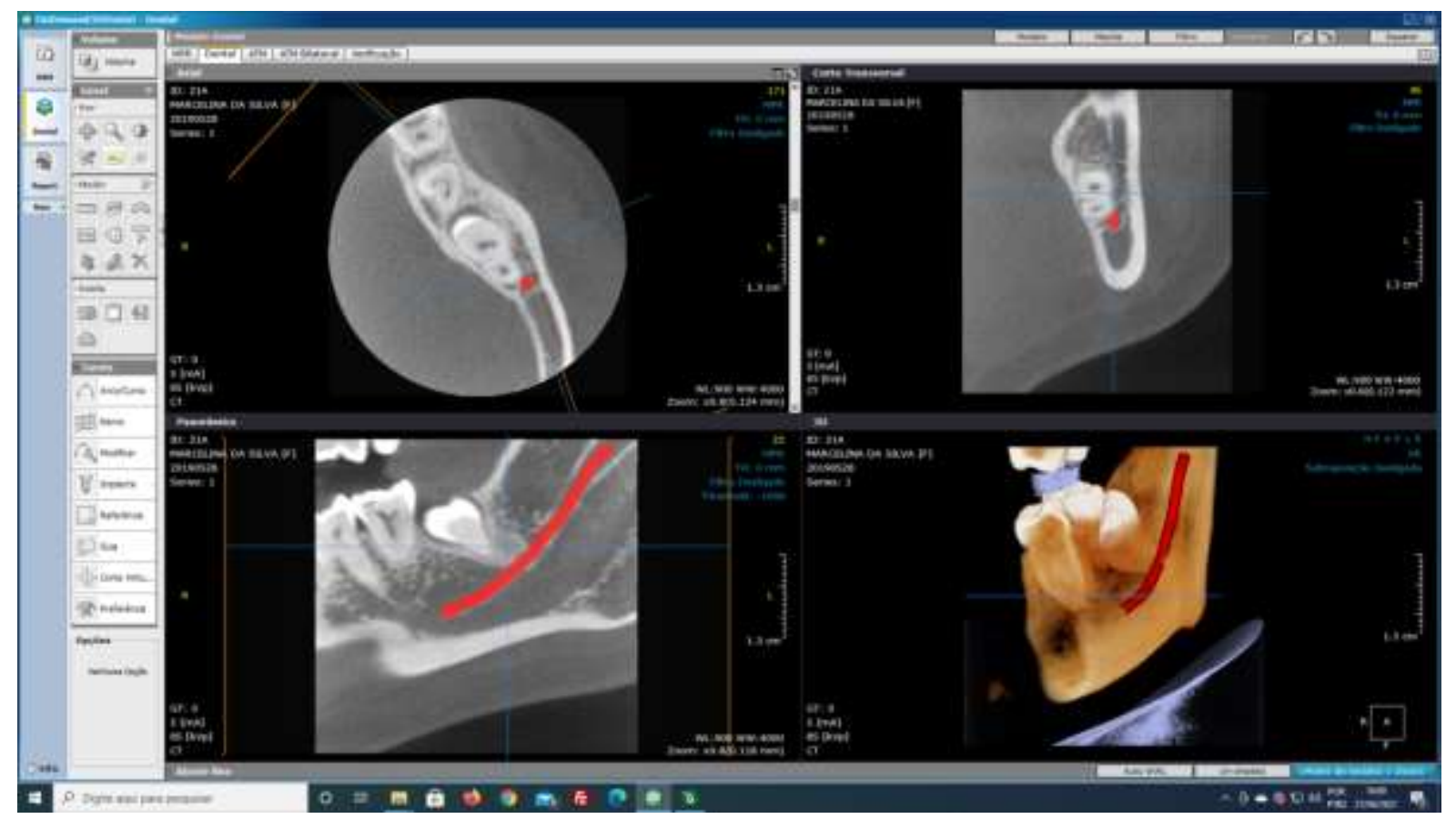

Fonte: Estomatologia UFC - Sobral.

Não podemos deixar de destacar ainda o grande número de exames que não apresentavam o campo de indicações preenchido tanto nas RPs quanto nas TCFCs, totalizando 131 exames e 30\% do total de exames realizados respectivamente. Esse fato é semelhante ao que ocorreu em um trabalho similar em uma universidade pública (Barros et al., 2015). A solicitação de exames é um documento de valor legal e jurídico e quando faltam informações abre espaço para problemas legais. É proibida toda exposição não justificada aos raios-X. Nesse caso faltaria a informação em questão entre outras como a quem foi entregue tal exame. O espaço em questão da atual folha de solicitação é insuficiente para justificar a solicitação conforme pode ser observado na Figura 5. Faltam também informações que seriam importantes para o tratamento como se trata de um exame de planejamento, acompanhamento etc. Por isso foi proposta a nova folha de solicitação que se encontra na Figura 6. 
Figura 5: Antiga folha de solicitação.

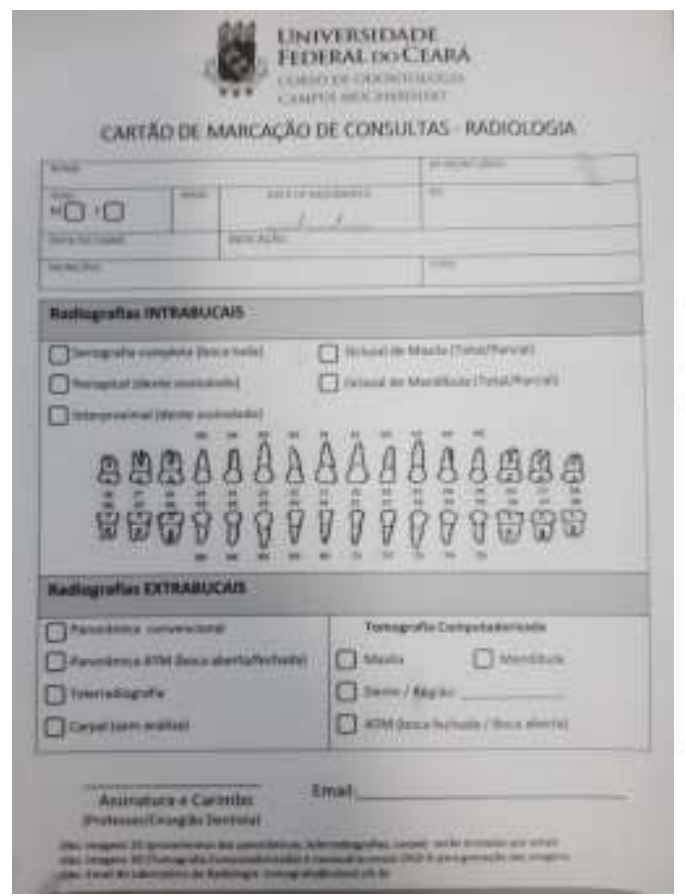

Figura 6: Nova folha de solicitação.

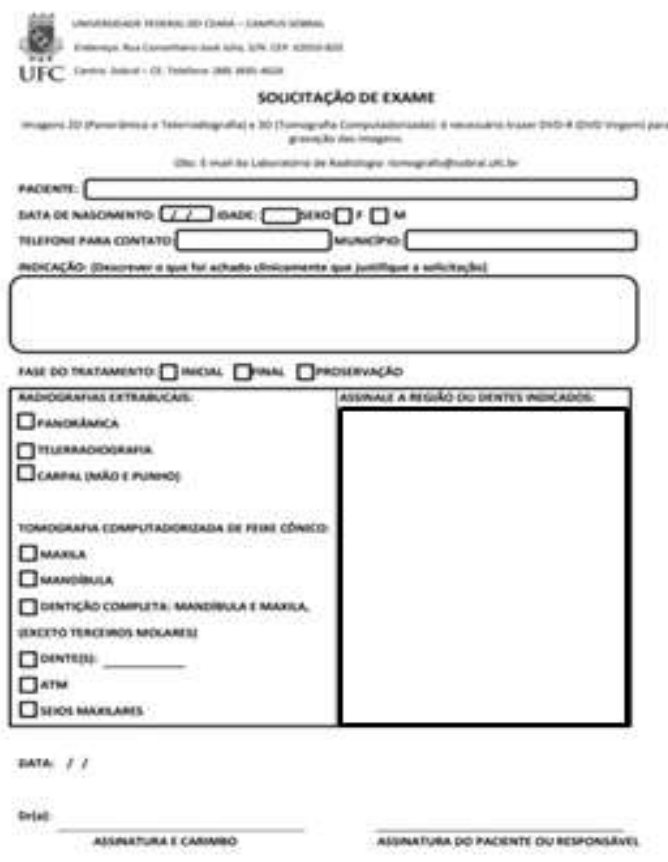

Fonte: Autores.

Independente do exame de imagem, ambas, Radiografia Panorâmica e Tomografia Computadorizada de feixe cônico, devem seguir o princípio ALARA (As Low As Reasonably Achievable) que segundo o site do Centro de Estudos Energéticos e Radiofísicos

[...] é um acrônimo para a expressão "tão baixo quanto razoavelmente exequível”. Este é um princípio de segurança de radiação, com o objetivo de minimizar as doses a pacientes e trabalhadores, empregando para tal todos os métodos razoáveis existentes.

Portanto, qualquer que seja a técnica escolhida para o caso em questão, deve sempre ser pesado se ela é realmente necessária para não haver exposição do paciente à uma dose de radiação desnecessária.

\section{Conclusão}

A maior parte dos exames foi corretamente indicada concordando com a literatura em questão tanto para as radiografias panorâmicas e tomografias computadorizadas de feixe cônico. Contudo, ainda falta nas folhas de solicitação disponíveis na Universidade informações que poderiam ajudar o técnico de radiologia, a saber melhor a região a ser radiografada por isso a importância do desenvolvimento de uma nova ficha de solicitação (Figura 6). Além disso, qualquer que seja a técnica escolhida para o caso em questão, deve sempre ser pesado se ela é realmente necessária para não expor o paciente à uma dose de radiação desnecessária.

Os exames imaginológicos são grandes auxiliares da prática clínica, mas o paciente, acima de tudo não pode ser submetido a esse tipo de análise sem a devida indicação ou mesmo faltando informações. Os dados aqui encontrados e agrupados podem servir de base para futuras pesquisas sobre o assunto, mas espera-se que desde já esse estudo provoque mudanças no preenchimento das solicitações e da sua importância como parte do tratamento do paciente. 


\section{Referências}

Almeida, L. H. S. de, Azevedo, M. S., Pappen, F. G., \& Romano, A. R. (2015). Hematomas de erupção: relato de três casos clínicos em bebês. Revista Da Faculdade de Odontologia - UPF, 20(2), 222-226. https://doi.org/10.5335/rfo.v20i2.4354

Alves, F. (2002). Avaliação da Qualidade Técnica e Interpretativa da Radiografia Panorâmica. 04.

Barros, M. C. S., Cral, W. G., Rubira-Bullen, I. R. F., \& Capelozza, A. L. A. (2015). Utilização e Vantagens da Tomografia Computadorizada por Feixe Cônico em Universidade Pública. APCD - Associação Paulista de Cirurgiões-Dentistas, 69(1168), 336-339.

Borges, C. L., \& Lobo, C. M. (2016). O Papel Dos Exames Bidimensionais E Da Tomografia Relação Entre Terceiros Molares Inferiores E Canal. Campinas, Universidade Estadual D E Piracicaba, Faculdade D E Odontologia D E.

Borsatto, M. C., \& Nelson-filho, P. (2007). Principais tumores odontogênicos que podem acometer a cavidade bucal de crianças. $19(2), 181-187$.

Carraro, G., \& Santos, F. C. (2014). A Importância da Tomografia Computadorizada para Avaliação de Áreas Edêntulas no Planejamento de Implantes. Journal of Oral Investigations, 3(2), 31-36. https://doi.org/10.18256/2238-510x/j.oralinvestigations.v3n2p31-36

da Silveira, K. G., Costa, F. W. G., Bezerra, M. F., Pimenta, A. V. de M., Carvalho, F. S. R., \& Soares, E. C. S. (2016). Sinais radiográficos preditivos de proximidade entre terceiro molar e canal mandibular através de tomografia computorizada. Revista Portuguesa de Estomatologia, Medicina Dentaria e Cirurgia Maxilofacial, 57(1), 30-37. https://doi.org/10.1016/j.rpemd.2015.11.006

Dalla, F., Mattiello, L., Lima, E. M. De, Maria, S., \& Rizzatto, D. (2016). Impacção De Incisivos Centrais Superiores : Etiologia e Tratamento Maxillary Central Incisor Impaction : Etiology and Treatment. Artigo de Revisao de Literatura, XXI(May 2018).

Estrela, C (2018). Metodologia científica: ciência, Ensino, pesquisa. (3a ed.), Artes Médicas, 1. 707p.

Freitas, A.; Rosa, J. E.; Souza, I. F. (2000). Radiologia Odontológica. (5a ed.), Artes Médicas.

Francisco, F. C., Maymone, W., Carlos, A., Carvalho, P., Frida, V., Francisco, M., \& Francisco, M. C. (2005). Radiologia: 110 anos de história. Medicina, 27(4), 281-286.

Garib, D. G., Raymundo Jr., R., Raymundo, M. V., Raymundo, D. V., \& Ferreira, S. N. (2007). Tomografia computadorizada de feixe cônico (Cone beam): entendendo este novo método de diagnóstico por imagem com promissora aplicabilidade na Ortodontia. Revista Dental Press de Ortodontia e Ortopedia Facial, 12(2), 139-156. https://doi.org/10.1590/s1415-54192007000200018

Gartner, C. F., \& Goldenberg, F. C. (2009). A Importância da Radiografia Panorâmica no Diagnóstico e no Plano de Tratamento Ortodôntico na Fase da Dentadura Mista. Odonto, 17(33), 102-109. https://doi.org/10.15603/2176-1000/odonto.v17n33p102-109

Ghaeminia, H., Meijer, G. J., Soehardi, A., Borstlap, W. A., Mulder, J., \& Bergé, S. J. (2009). Position of the impacted third molar in relation to the mandibular canal. Diagnostic accuracy of cone beam computed tomography compared with panoramic radiography. International Journal of Oral and Maxillofacial Surgery, 38(9), 964-971. https://doi.org/10.1016/j.ijom.2009.06.007

Ghaeminia, H., Meijer, G. J., Soehardi, A., Borstlap, W. A., Mulder, J., Vlijmen, O. J. C., Bergé, S. J., \& Maal, T. J. J. (2011). The use of cone beam CT for the removal of wisdom teeth changes the surgical approach compared with panoramic radiography: A pilot study. International Journal of Oral and Maxillofacial Surgery, 40(8), 834-839. https://doi.org/10.1016/j.ijom.2011.02.032

Guilherme, I., Santos, P., Camyla, L., \& Gomes, P. (2016). Artigo Original Topografia do Canal Mandibular e Relação com Terceiros Molares em Tomografias por Feixe Cônico. 5458, 12-17.

Gustavo, M., Rodrigues, S., Martín, O., Alarcón, V., Carraro, E., Rocha, J. F., Lúcia, A., \& Capelozza, Á. (2010). Tomografia computadorizada por feixe cônico : formação da imagem, indicações e critérios para prescrição Cone-beam computed tomography: Formation of the image, indications and selection criteria. Odontol Clín Cient, 9(2), 115-118.

Haas, L. F. (2013). Reconstrução Panorâmica X Radiografia Panorâmica: Revisão de Literatura. 2013. 10f. Monografia - Curso de Especialização e Radiologia Odontológica e Imaginologia pela Faculdade de Odontologia da Universidade Federal do Rio Grande do Sul, Porto Alegre.

Jelodar, S., Ghadirian, H., Ketabchi, M., Ahmadi Karvigh, S., \& Alimohamadi, M. (2018). Bilateral Ischemic Stroke Due to Carotid Artery Compression by Abnormally Elongated Styloid Process at Both Sides: A Case Report. Journal of Stroke and Cerebrovascular Diseases, 27(6), e89-e91. https://doi.org/10.1016/j.jstrokecerebrovasdis.2017.12.018

Kato, R. B., Bueno, R. B. L., Neto, P. J. de O., Ribeiro, M. C., \& Azenha, M. R. (2016). Acidentes e Complicações Associadas à Cirurgi dos Terceiros Molares Realizada por Alunos de Odontologia. Dentomaxillofacial Radiology, 10(1), 45-54. https://doi.org/10.1016/j.jcms.2016.07.025

Mozzo, P., Procacci, C., Tacconi, A., Martini, P. T., \& Andreis, I. A. (1998). A new volumetric CT machine for dental imaging based on the cone-beam technique: preliminary results. European radiology, 8(9), 1558-1564. https://doi.org/10.1007/s003300050586

Nonato, M. D. M. (2006). Aspectos Relacionados ao Emprego da Radiografia Panorâmica em Pacientes Infantis Aspects Related To the Use of Panoramic Radiography in Children. 15-19.

Nunes, C. E. N., Mourão, A. C. C. M., Sampieri, M. B. da S., Oliveira, D. H. I. P. de, \& Chaves, F. N. (n.d.). Using digital panoramic radiographs to examine temporal styloid process elongation.

Pippi, R., Santoro, M., \& D’Ambrosio, F. (2016). Accuracy of cone-beam computed tomography in defining spatial relationships between third molar roots and inferior alveolar nerve. European Journal of Dentistry, 10(4), 454-458. https://doi.org/10.4103/1305-7456.195168 
Research, Society and Development, v. 10, n. 10, e291101018778, 2021

(CC BY 4.0) | ISSN 2525-3409 | DOI: http://dx.doi.org/10.33448/rsd-v10i10.18778

Pozzer, L., Jaimes, M., Chaves Netto, H. D. de M., Olate, S., \& Barbosa, J. R. de A. (2009). Cistos odontogênicos em crianças: análise da descompressão cirúrgica em dois casos TT - The odontogenic cyst in children: analysis of the surgical decompression in 2 cases. Rev. Cir. Traumatol. Buco-Maxilo-Fac, 9(2), 17-22. http://www.revistacirurgiabmf.com/2009/v9n2/02.pdf

Ribeiro, S. (2016). Princípio Alara. Centro de Estudos Energéticos e Radiofísicos. https://www.ceer.es/pt-pt/principio-alara/

Silva, N. R. de A., \& Passos, A. G. (2014). Radiografia Panorâmica Para Extração Dos Terceiros Molares.

Suomalainen, A., Ventä, I., Mattila, M., Turtola, L., Vehmas, T., \& Peltola, J. S. (2010). Reliability of CBCT and other radiographic methods in preoperative evaluation of lower third molars. Oral Surgery, Oral Medicine, Oral Pathology, Oral Radiology and Endodontology, 109(2), 276-284. https://doi.org/10.1016/j.tripleo.2009.10.021

Tavano, O., \& Alvarez, L. C. (2002). Curso de Radiologia em Odontologia. (4a ed.), Liv. Santos.

Whaites, E. (2009). Princípios de radiologia odontológica. (4a ed.), Elsevier.

White, S. C., \& Pharoah, M. J. (2004). Radiologia Oral. (5a ed.), Mosby. 